\title{
Preferential Adsorption in Ethane/Carbon Dioxide Fluid Mixtures Confined within Silica Nanopores
}

\author{
M. Dolores Elola* ${ }^{* \dagger}$ a and Javier Rodriguez ${ }^{\dagger, \dagger}$ \\ ${ }^{\dagger}$ Departamento de Física, Comisión Nacional de Energía Atómica, Avenida Libertador 8250, 1429 Buenos Aires, Argentina \\ ${ }^{\ddagger}$ ECyT, UNSAM, Martín de Irigoyen 3100, 1650 San Martín, Provincia de Buenos Aires, Argentina
}

Supporting Information

\begin{abstract}
Molecular dynamics simulations have been performed to investigate the structural and dynamical properties of fluid ethane confined within cylindrical silica nanopores of 3.8 and $1 \mathrm{~nm}$ diameters. Pure ethane and equimolar mixtures of ethane and $\mathrm{CO}_{2}$ were considered for the analysis. Computer simulations were carried out along the supercritical isotherm $T=320 \mathrm{~K}$, exploring densities within the range $\rho / \rho_{\mathrm{c}}=0.05-2.28$. Density profiles along the axial and radial directions of the pore, orientational distribution functions, analysis of interfacial dynamic properties, and estimations of diffusion coefficients are presented. In agreement with experimental data, the results show the formation of a dense adsorption layer, with densities that may be up to 10 times larger

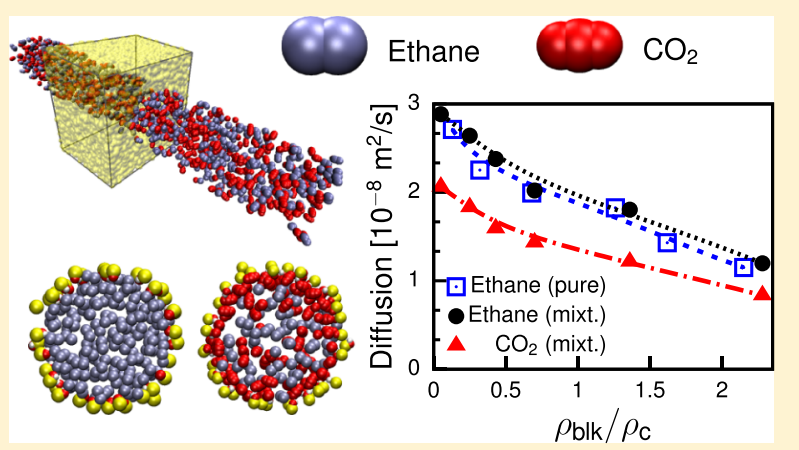
than those of the bulk phase, in particular at low densities and under subnanometer confinement. The incorporation of $\mathrm{CO}_{2}$ changes the scenario, leading to preferential adsorption of $\mathrm{CO}_{2}$ over ethane species. The dynamics of ethane also change in mixtures, in agreement with experimental measurements. These changes are manifested by increments in the diffusion coefficients of confined ethane in the presence of $\mathrm{CO}_{2}$. However, in contrast with experimental data, our results for the diffusion coefficients of confined ethane showed a monotonic decreasing behavior with increasing bulk density. Plausible interpretations for this discrepancy are also discussed.
\end{abstract}

\section{INTRODUCTION}

Fluids under nanometric confinement are relevant to a diverse variety of chemical systems, as they may exhibit distinctive characteristics that differ from those observed in bulk phases. In particular, when the fluid is confined within nanometer length scales, between 1 and $10 \mathrm{~nm}$, an important fraction of the fluid molecules are restricted to be at the interfacial layer. As such, the resulting properties will be strongly influenced not only by the size, shape, and topology of confinement, but also by the interplay between liquid-liquid and liquid-surface interactions, and entropic confinement effects as well. A physical interpretation of these modifications is then essential in order to understand the onset of these modified properties of the confined fluids.

In particular, the investigation of the properties of $\mathrm{CO}_{2}$ and hydrocarbons trapped within cavities of nanometer dimensions has received great attention in the last years. ${ }^{1-8}$ The study of these systems is relevant, for instance, for carbon storage technologies and shale gas production, among other applications. Shale gas production is based on fracturing technologies, leading to the extraction of the natural gas from the shale formations, where the gas is stored. The fracturing process consists in injecting a fluid at pressures high enough to fracture the rock, and thereby, increase the permeability to allow the gas extraction. In addition to methane, ethane is one of the fluids that can be recovered from shale reservoirs at a relatively large scale. Because of its low cost, water is the most regularly used fracking fluid in commercial shale gas production. A microscopic analysis of the molecular details of $\mathrm{CO}_{2}$-pore and ethane-pore interactions becomes then critical for a serious evaluation of $\mathrm{CO}_{2}$ as an alternative fracking fluid.

The properties of confined fluid phases are usually studied by diverse experimental methodologies, such as neutron scattering, ${ }^{9,10}$ quasielastic neutron scattering, ${ }^{11}$ gravimetry, ${ }^{3,12}$ and volumetric sorption techniques. ${ }^{13,14}$ More specifically, Patankar et al. ${ }^{15}$ have recently investigated the role of confinement on adsorption and dynamics of ethane and ethane $/ \mathrm{CO}_{2}$ mixtures in mesoporous controlled pore glass systems, by gravimetric adsorption and quasielastic neutron scattering techniques. Their measurements of self-diffusion coefficients for confined ethane yielded values that were nearly four times smaller than the bulk-like ones, at $T=323 \mathrm{~K}$ and in a pressure range between 38 and 80 bar. The experimental dependence of the confined ethane diffusivity on the bulk density shows a minimum near the supercritical density. Above that value, increments of the diffusion of confined ethane was observed as the bulk density is further incremented. This

Received: August 21, 2019

Revised: December 2, 2019

Published: December 2, 2019 
behavior is interpreted by the authors in terms of the larger fraction of fluid-fluid interactions (as opposed to fluid/wall interactions) that dominate the fluid dynamics under those conditions. The addition of $\mathrm{CO}_{2}(27 \% \mathrm{~mol})$ led to a significant increment of the ethane mobility, most likely because of the displacement of interfacial ethane molecules by $\mathrm{CO}_{2}$ species.

Structural and dynamical features of simple fluids, such as argon, carbon dioxide, and $\mathrm{H}_{2}$, close to silica surfaces have been also investigated by computer simulations in recent years. ${ }^{16-21}$ More recent works also include the study of propane, $n$-butane, and $\mathrm{CO}_{2} / n$-butane mixtures under slitshaped confinement. ${ }^{22,23}$ A comparative study of several properties of the pure phases of ethane and $\mathrm{CO}_{2}$ fluids confined in silicalite zeolites has been performed at high pressures (100 bar) using a combination of Monte Carlo and molecular dynamics (MD) simulations. ${ }^{24}$ Specifically, the authors found a faster diffusivity of $\mathrm{CO}_{2}$ at low pressures, whereas at high pressures, a slower diffusion of $\mathrm{CO}_{2}$ as compared to that of ethane was reported. Rotational motions of ethane exhibited shorter timescales than $\mathrm{CO}_{2}$. These differences were rationalized by the authors as a consequence of the interplay between the molecular kinetic diameter and the quadrupole moment of $\mathrm{CO}_{2}$ and ethane interactions.

Carbon dioxide is a linear symmetric molecule with no net dipole moment, and a permanent electrical quadrupole moment that can be described as two electrical dipoles sitting back-to-back, pointing in opposite directions. Ethane, on the other hand, is also a linear symmetric molecule with no dipole moment, but with a quadrupole moment that is about five times smaller than that of $\mathrm{CO}_{2} \cdot{ }^{25,26}$ This difference in the quadrupole moment has been suggested as a key feature, responsible for the very different behavior of $\mathrm{CO}_{2}$ and ethane species when a fluid mixture of them is confined within silica cavities. In previous work, we studied the adsorption of $\mathrm{CO}_{2}$ within cylindrical silica pores, along a supercritical isotherm, by computer simulations. ${ }^{27}$ As an extension of that early work, and largely motivated by the results reported by Patankar et al., ${ }^{15}$ in this work we explore the behavior of fluid ethane and ethane $/ \mathrm{CO}_{2}$ equimolar mixtures confined within cylindrical silica nanopores of 3.8 and $1 \mathrm{~nm}$ diameters, by performing $\mathrm{MD}$ simulations. Usually, the experiments are able to measure the average fluid density within the pore cavity; however, information about the local density relative to the pore walls cannot be accessed from neutron scattering or gravimetric measurements. Therefore, we have attempted to perform a structural and dynamical characterization of the fluid confined within silica nanopores, at a microscopic level.

The paper is organized as follows: in Section 2, we describe the systems studied and the potential models, along with simulations details. In Section 3, we present and discuss the results obtained for the structural and dynamical properties. We conclude in Section 4 with a summary of our main findings.

\section{MODEL AND SIMULATION DETAILS}

We carried out MD simulations of systems composed of cylindrical pores with diameters $D_{\mathrm{p}}=1$ and $3.8 \mathrm{~nm}$, filled with pure ethane and equimolar mixtures of ethane and $\mathrm{CO}_{2}$, at supercritical conditions. All different components of the simulated systems were modeled as a collection of interacting sites: the overall potential energy was decomposed into a sum of site-site, pairwise interactions combining dispersion (Lennard-Jones) and Coulomb contributions. Inter- and intramolecular interactions in ethane were modeled by the transferable potential for phase equilibria called UA2 (unitedatom approach). ${ }^{28}$ Computer simulations using this potential model predict ethane critical properties of $\rho_{\mathrm{c}}^{\mathrm{UA} 2}=0.214 \mathrm{~g} \mathrm{~cm}^{-3}$ and $T_{\mathrm{c}}^{\mathrm{UA} 2}=308 \mathrm{~K}($ ref 28$)$, to be compared with the experimental values: $\rho_{\mathrm{c}}=0.207 \mathrm{~g} \mathrm{~cm}^{-3}$ and $T_{\mathrm{c}}=305 \mathrm{~K}$ (ref 29). All simulated thermodynamic states were chosen along the $T=320 \mathrm{~K}$ isotherm, covering a range of $\mathrm{C}_{2} \mathrm{H}_{6}$ densities spanning between $\rho / \rho_{\mathrm{c}}=0.13$ and 2.15. The two-site Elementary Physical Model (EPM2) potential ${ }^{30}$ was employed to model intermolecular interactions in $\mathrm{CO}_{2}$. In ref 30, the authors adjusted the EPM force field parameters so as to accurately reproduce critical constants, obtaining $\rho_{\mathrm{c}}^{\mathrm{EPM} 2}=0.45$ $\mathrm{g} \mathrm{cm}^{-3}$ and $T_{\mathrm{c}}^{\mathrm{EPM} 2}=313 \mathrm{~K}$; whereas the experimental reported values are $\rho_{\mathrm{c}}=0.47 \mathrm{~g} \mathrm{~cm}^{-3}$ and $T_{\mathrm{c}}=304 \mathrm{~K}$. The EPM2 force field has then been taken from ref 30 and used in this work without any additional modification.

For the case of $\mathrm{C}_{2} \mathrm{H}_{6}-\mathrm{CO}_{2}$ mixtures, the critical parameters were obtained from the analytic interpolation equations reported in ref 31 . The investigated densities of the mixtures were in the range $\rho / \rho_{\mathrm{c}}=0.05$ to 2.28 .

Force field parameters for silica were taken from ref 32 . As such, cross interactions between the pore and the fluid phase were computed assuming the usual arithmetic and geometric means for length and energy parameters, respectively.

The preparation of the systems involved a sequential procedure. First, a cylindrical silica pore of diameter $D_{\mathrm{p}}=$ 3.8 $\AA$ was built up from an amorphous silica block of dimensions $L_{x}=L_{y}=70 \AA, L_{z \mathrm{p}}=53 \AA$. The silica block to generate the small pore with $D_{\mathrm{p}}=1 \mathrm{~nm}$ had dimensions $L_{x}=$ $L_{y}=30 \AA, L_{z p}=53 \AA$. Details of this procedure can be found in previous works. ${ }^{33,34}$ In order to mimic the experimental setup of hydroxylated porous glasses, hydrophilic characteristics were provided to the pore walls: the unsaturated oxygens at the internal walls of the pore were fully protonated, yielding a density of $\mathrm{SiOH}$ groups of $\approx 3 \mathrm{~nm}^{-2}$, in reasonable agreement with data obtained from gas adsorption experiments. ${ }^{35}$

The final stage involved the incorporation of the fluid phase within the pores. To do so, the interiors of the pores were filled with the fluid molecules, and two adjacent bulk-like fluid reservoirs of dimensions $L_{x} \times L_{y} \times 100 \AA$ were brought in contact with the silica block at the pore sides. The overlapping molecules were discarded. From then on, the fluid phase was allowed to re-equilibrate at $T=320 \mathrm{~K}$ for about $5 \mathrm{~ns}$. During this period, a Langevin thermostat was employed, set at $T=$ $320 \mathrm{~K}$, with a damping constant of 1 ps. This thermostat was combined with a Nosé-Hoover Langevin piston to control the pressure at the desired value. The barostat oscillation period and the damping time scale were fixed at the typically used values of 200 and $100 \mathrm{fs}$, respectively. The size of the simulation box was dynamically adjusted only in the $z$ direction, keeping the dimensions of the unit cell constant in the $x y$ plane Therefore, during the equilibration period, the length of the simulation box along the $z$-axis was adjusted to set the pressure and local density at the center of the external reservoirs similar to those of the corresponding bulk values. This is done to ensure the formation of an equilibrated bulk fluid phase. Equilibrium was considered achieved when the fluid densities reached constant values and the thermal and energy fluctuations remained within $10 \%$ of their average values. Figure 1 shows snapshots of typical configurations captured during the MD simulations. From the bulk density $\rho_{\mathrm{b}}$ at a given temperature, the corresponding bulk pressure was 


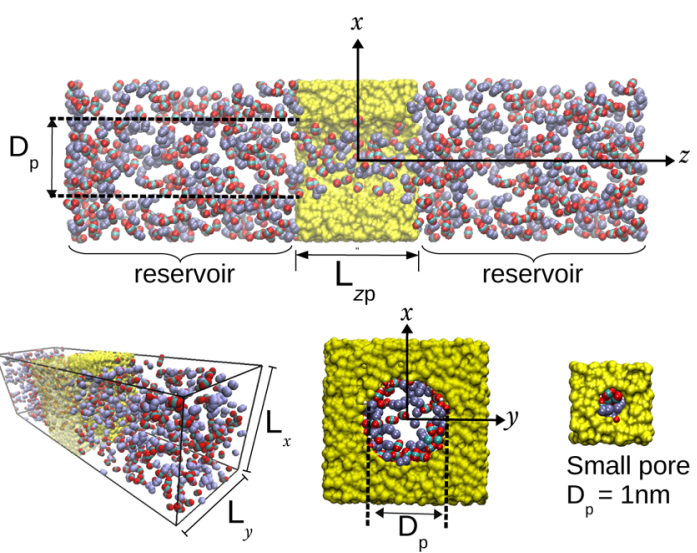

Figure 1. Typical snapshots from different views of the configuration of the larger pore system, showing the reference axes and the fluid reservoirs in contact with the silica pore.

obtained from the experimental isothermal properties of the NIST database. ${ }^{36}$ The effect of adding $\mathrm{CO}_{2}$ on the structure and dynamics of ethane was evaluated by investigating solely equimolar mixtures of $\mathrm{C}_{2} \mathrm{H}_{6} / \mathrm{CO}_{2}$. The preparation of these mixed systems followed the same steps as those described above for the pure- $\mathrm{C}_{2} \mathrm{H}_{6}$ ones. The interiors of the pores were initially filled with an equimolar fluid mixture of $\mathrm{C}_{2} \mathrm{H}_{6}$ and $\mathrm{CO}_{2}$. Likewise, the two adjacent bulk-like reservoirs contained a fluid mixture with equal numbers of $\mathrm{C}_{2} \mathrm{H}_{6}$ and $\mathrm{CO}_{2}$ molecules. After a $\sim 5 \mathrm{~ns}$ equilibration period, $\mathrm{MD}$ trajectories of length $50 \mathrm{~ns}$ were saved for data analysis. The total numbers of molecules considered in each simulated system are listed in Table 1.

All MD trajectories were generated using the NAMD software $^{37}$ and corresponded to microcanonical runs. Periodic boundary conditions were applied along the three Cartesian coordinates. Short-ranged intermolecular forces were cut off at $14 \AA$, whereas the particle mesh Ewald method was implemented to handle long-range Coulomb forces. ${ }^{38,39}$ The equations of motion were integrated using a velocity form of the Verlet algorithm, with a multiple time step integration scheme, known as impulse-based Verlet-I, ${ }^{40,41}$ with a time step of $1 \mathrm{fs}$ for intramolecular modes and nonbonded short-ranged forces, and $2 \mathrm{fs}$ for the rest of the Coulomb forces.

\section{RESULTS}

Unless explicitly mentioned, from now on, we will refer to the results obtained in the wider pore.

3.1. Structural Properties. We start our analysis by investigating the structural features of the fluid within the pores. To that aim, we will explore the local density profiles of $\mathrm{C}_{2} \mathrm{H}_{6}$ along the longitudinal axis, computed as

$$
\rho(z)=\frac{1}{\pi R_{\mathrm{p}}^{2}} \sum_{i}\left\langle\delta\left(z_{i}-z\right)\right\rangle_{R_{\mathrm{p}}}
$$

where $z_{i}$ is the $z$-coordinate of the center-of-mass of the $i$-th molecule and $\langle\cdots\rangle_{R_{\mathrm{p}}}$ denotes an average restricted to those molecules with distances to the $z$-axis less than $R_{\mathrm{p}}=D_{\mathrm{p}} / 2$. The profiles of pure ethane systems are shown in Figure 2. Note

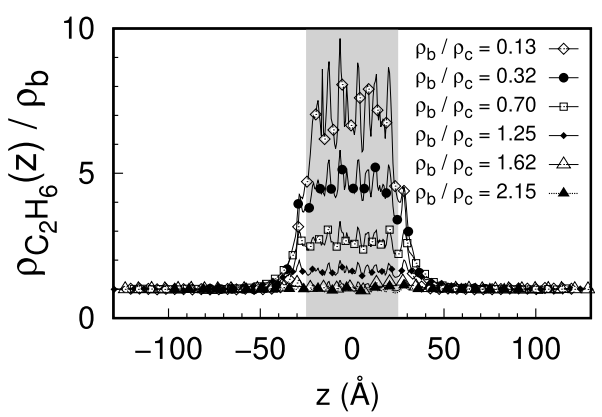

Figure 2. Local densities of ethane (normalized to $\rho_{\mathrm{b}}$ ) along the $z$ axis, for pure ethane confined within the simulated pores. Densities have been computed within an axial cylinder of diameter $D_{\mathrm{p}}=3.8 \mathrm{~nm}$ and length $L_{z}$. The shaded region indicates the extension of the silica pore.

Table 1. Details of the Simulations ${ }^{a}$

\begin{tabular}{|c|c|c|c|c|c|c|c|c|}
\hline system & $P$ (bar) & $\rho_{\mathrm{b}}\left(\mathrm{g} \mathrm{cm}^{-3}\right)$ & $\rho_{\mathrm{b}} / \rho_{\mathrm{c}}$ & $N_{\mathrm{C}_{2} \mathrm{H}_{6}}$ & $N_{\mathrm{CO}_{2}}$ & $\left\langle N_{\mathrm{C}_{2} \mathrm{H}_{6}}\right\rangle_{\mathrm{p}}$ & $\left\langle N_{\mathrm{CO}_{2}}\right\rangle_{\mathrm{p}}$ & $\left\langle x_{\mathrm{C}_{2} \mathrm{H}_{6}}\right\rangle_{\mathrm{p}}$ \\
\hline Eth-20 & 20 & 0.026 & 0.13 & 950 & & 207 & & 1.00 \\
\hline Eth-40 & 40 & 0.064 & 0.32 & 1332 & & 313 & & 1.00 \\
\hline eth-40 & & & & 370 & & 40 & & 1.00 \\
\hline Eth-57 & 57 & 0.139 & 0.70 & 2960 & & 353 & & 1.00 \\
\hline Eth-70 & 70 & 0.256 & 1.26 & 5322 & & 373 & & 1.00 \\
\hline Eth-100 & 100 & 0.328 & 1.62 & 6825 & & 387 & & 1.00 \\
\hline eth-100 & & & & 1230 & & 45 & & 1.00 \\
\hline Eth-400 & 400 & 0.438 & 2.15 & 6440 & & 440 & & 1.00 \\
\hline Mix-20 & 20 & 0.031 & 0.05 & 500 & 500 & 91 & 124 & 0.42 \\
\hline Mix-40 & 40 & 0.072 & 0.25 & 618 & 618 & 137 & 195 & 0.41 \\
\hline $\operatorname{mix}-40$ & & & & 205 & 205 & 29 & 20 & 0.41 \\
\hline Mix-57 & 57 & 0.137 & 0.46 & 1144 & 1144 & 162 & 230 & 0.41 \\
\hline Mix-70 & 70 & 0.217 & 0.70 & 1726 & 1726 & 177 & 251 & 0.41 \\
\hline Mix-100 & 100 & 0.394 & 1.35 & 3363 & 3363 & 183 & 300 & 0.38 \\
\hline $\operatorname{mix}-100$ & & & & 720 & 720 & 36 & 19 & 0.35 \\
\hline Mix-400 & 400 & 0.685 & 2.28 & 3820 & 3820 & 213 & 344 & 0.38 \\
\hline
\end{tabular}

${ }^{a} \rho_{\mathrm{b}}$ corresponds to the bulk density at the lateral reservoirs, $P$ represents the corresponding pressure at $T=320 \mathrm{~K} . N_{\mathrm{C}_{2} \mathrm{H}_{6}}$ and $N_{\mathrm{CO}_{2}}$ correspond to the total numbers of ethane and carbon dioxide molecules; angular brackets $\langle\cdots\rangle_{\mathrm{p}}$ indicate the average inside the silica pore. "Eth" and "Mix" labels correspond to the wider pore systems $\left(D_{\mathrm{p}}=3.8 \mathrm{~nm}\right)$; "eth" and "mix" ones, to the smaller pore of $1 \mathrm{~nm}$ diameter. 
that, at sufficiently long distances away from the pore openings, that is, $|z| \gtrsim 60 \AA$, all density profiles reach their corresponding bulk values $\left(\rho_{\mathrm{b}}\right)$. However, within the interior of the pore, a density enhancement is clearly perceptible. Moreover, the relative increment looks much more pronounced at low bulk densities, attaining $\rho / \rho_{\mathrm{b}} \approx 5$ for $\rho_{\mathrm{b}} / \rho_{\mathrm{c}}=0.32$. This relative increment is further enhanced within the smaller pores. In fact, $\rho / \rho_{\mathrm{b}}$ rises up to values of $\sim 10$ when the pore diameter is reduced to $1 \mathrm{~nm}$ (see Figure S1 in the Supporting Information section). Similar density enhancements have been found for confined $\mathrm{CO}_{2}$ within silica pores of comparable sizes. ${ }^{4,5,27}$ As shown in Figure S2 of the Supporting Information section, it is important to note that the absolute densities of confined ethane seem to be independent of the external bulk density.

The changes in the $\mathrm{C}_{2} \mathrm{H}_{6}$ structure upon addition of $\mathrm{CO}_{2}$ can be observed in Figure 3. The densities of the simulated

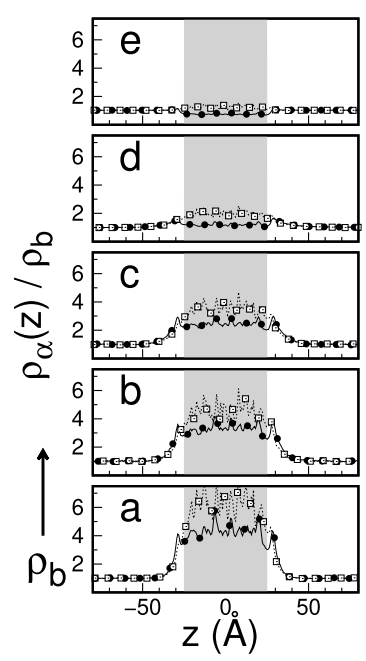

Figure 3. Normalized local densities of ethane $(-\bullet-)$ and $\mathrm{CO}_{2}$ $(\cdots \square \cdots)$ species along the axial $z$ axis, for ethane $/ \mathrm{CO}_{2}$ equimolar mixtures confined within the simulated pores. Panels (a-e) correspond to $\rho / \rho_{\mathrm{c}}=0.25,0.46,0.70,1.35$, and 2.28 , respectively. The shaded region indicates the extension of the silica pore.

systems were in the range $\rho_{\mathrm{b}} / \rho_{\mathrm{c}}=0.25-2.28$. The profiles reveal that, although all MD simulations were started from configurations corresponding to exact equimolar compositions, silica pore walls promote a preferential density enhancement of $\mathrm{CO}_{2}$ species over $\mathrm{C}_{2} \mathrm{H}_{6}$ ones within the pores. This preferential adsorption effect could be mainly ascribed to the polar interactions between $\mathrm{CO}_{2}$ and the surface $\mathrm{SiOH}$ groups. Consequently, the effective average ethane molar fraction within the pore resulted in all cases being below 0.5 (see last column of Table 1). It is also worth noting that, at the highest simulated density, shown in Figure 3e, the $\mathrm{CO}_{2}$ species inside the pore expel ethane molecules toward outside the cavity, leading to an $\mathrm{C}_{2} \mathrm{H}_{6}$ confined density that is smaller than that of the bulk phase, probably because of steric restrictions arising from inefficient packing as compared to the bulk mixture.

Quantitative complementary information about how the density profiles look like along the cylindrical radial direction inside the pores can be extracted by computing local density fluctuations along radial directions

$$
\rho(r)=\frac{1}{2 \pi r L_{z \mathrm{p}}} \sum_{i}\left\langle\delta\left(r_{i}-r\right)\right\rangle
$$

where $r_{i}$ corresponds to the distance between the center-ofmass of the $i$-th molecule and the pore axis. In Figure 4, we

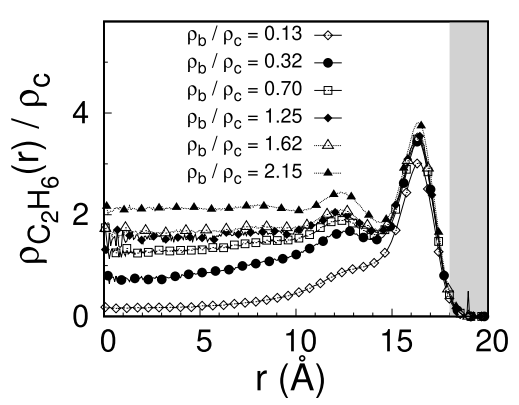

Figure 4. Local densities of ethane (normalized to $\rho_{\mathrm{c}}$ ) along the cylindrical radial direction, for pure ethane confined within the simulated pores. The shaded region represents the location of the silica walls.

present results for radial densities of pure ethane systems. The profiles reveal the presence of a strongly adsorbed interfacial layer, whose maxima are located approximately $2 \AA$ inward from the pore wall. The shape and magnitude of the adsorbed peak exhibit practically no change, as the bulk density experiences a sevenfold increment. More precisely, note that the local densities of the adsorbed layers are comparable to or even larger than typical density values of $\mathrm{C}_{2} \mathrm{H}_{6}$ liquid phases near the condensation point at $1 \mathrm{~atm}, \rho_{\mathrm{cp}} / \rho_{\mathrm{c}} \approx 3$. These values suggest a surface-induced condensation phenomenon, characterized by a highly stable adsorbed layer. In addition to the adsorbed phase, the profiles of Figure 4 show adjacent layers, of smaller amplitude, located $\sim 6 \AA$ inward from the pore wall. Moreover, the magnitude of these local fluctuations increases as the bulk density is incremented.

The changes that occurred in the adsorption layer because of addition of $\mathrm{CO}_{2}$ are shown in Figure 5, where the radial

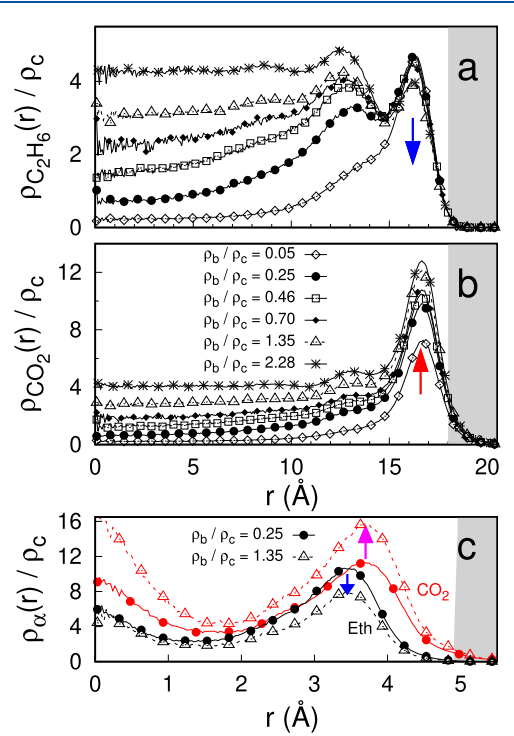

Figure 5. Normalized radial densities of (a) ethane and (b) $\mathrm{CO}_{2}$ species along the cylindrical radial direction, for ethane $/ \mathrm{CO}_{2}$ mixtures confined within the wider pores. Panel (c) shows the radial profiles obtained for the mixtures in the smaller pores (black and red lines correspond to ethane and $\mathrm{CO}_{2}$ species, respectively). The arrows indicate the trend in the peak intensity of the adsorbed layer when the bulk density is incremented. 
density profiles for $\mathrm{C}_{2} \mathrm{H}_{6}$ and $\mathrm{CO}_{2}$ species in confined equimolar mixtures are displayed. First, we note an overall $\sim 3$-fold increment in the magnitude of the maximum density of the adsorbed layer of $\mathrm{CO}_{2}$ when compared to that of $\mathrm{C}_{2} \mathrm{H}_{6}$ species. Second, the values of the maximum adsorption peaks for $\mathrm{CO}_{2}$ and $\mathrm{C}_{2} \mathrm{H}_{6}$ species exhibit opposite trends upon bulk density increments, reflecting $\mathrm{CO}_{2}$ preferential adsorption: whereas the $\mathrm{CO}_{2}$ adsorbed density increases with larger bulk densities, that of $\mathrm{C}_{2} \mathrm{H}_{6}$ decreases. More specifically, note that at the highest $\rho_{\mathrm{b}}$ considered, the ethane density near the interface is even smaller than at the center of the pore. As a result, although the global molar fraction of $\mathrm{C}_{2} \mathrm{H}_{6}$ inside the pore is about 0.40 , it is substantially smaller in the vicinity of the silica walls.

The densities along the radial coordinate in the smaller pores are displayed in Figure $5 \mathrm{c}$, where the preferential $\mathrm{CO}_{2}$ adsorption is again perceivable: note the larger magnitude of the density associated to $\mathrm{CO}_{2}$ species near the pore surfaces, along with a closer location of these peaks with respect to the pore surface, in comparison with those corresponding to ethane species. On comparison with the profiles in the wider pores, a similar trend is observed upon bulk density increments: the $\mathrm{C}_{2} \mathrm{H}_{6}$ adsorbed density decreases at the expense of an increment in that of $\mathrm{CO}_{2}$ species, which exhibits states of high density also at the center of the pore. In passing, we notice that the fluids in the smaller pore are characterized by an adsorbed layer that is even more dense than those in the wider pores. This result has already been found in previous works, and goes in line with the idea of greater efficiency of small subnanometer pores over larger cavities as adsorption devices. ${ }^{4,5,9,10,27}$

The computation of local mole fractions of ethane and $\mathrm{CO}_{2}$ species as a function of the radial coordinate, displayed in Figure 6, quantitatively confirm the $\mathrm{CO}_{2}$ enrichment of the

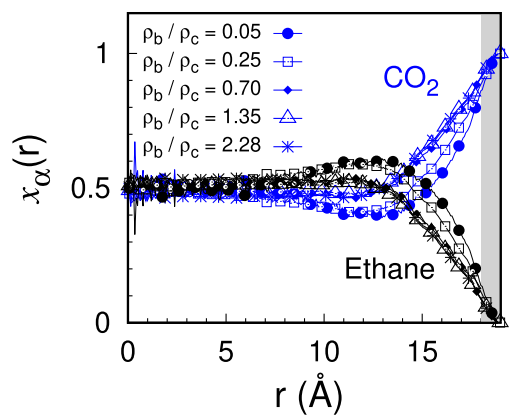

Figure 6. Local mole fractions of confined ethane (black) and $\mathrm{CO}_{2}$ (blue) species as a function of the radial coordinate, for equimolar mixtures at different bulk densities.

mixture within the first adsorption layer $(r \geq 15.5 \AA)$. The local mole fractions also show that the second layer $(10 \leq 15$ $\AA$ ) contains a larger fraction of ethane than $\mathrm{CO}_{2}$ species, whereas near the center of the pore, within the bulk-like region ( $r \leq 8 \AA$ ), an equimolar composition can be recovered.

The global sorption efficiency can be gauged from quantitative grounds by computing the excess densities, namely, $\Delta \rho_{\text {exc }}=\left\langle\rho_{\mathrm{p}}\right\rangle-\rho_{\mathrm{b}}$, as the difference between $\left\langle\rho_{\mathrm{p}}\right\rangle$, the average density inside the pore, and the bulk density. The results for the excess densities in the pure systems are displayed in Figure 7. In agreement with experimental data, the curves exhibit a maximum below $\rho_{\mathrm{b}} / \rho_{\mathrm{c}} \approx 0.5$, followed by a smooth drop as the bulk density increases. At high bulk

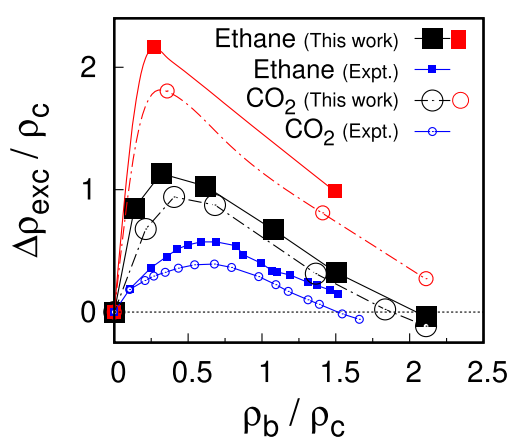

Figure 7. Excess densities of confined $\mathrm{CO}_{2}$ and $\mathrm{C}_{2} \mathrm{H}_{6}$ in the corresponding pure fluids. Experimental data (blue symbols) have been taken from ref 15 , measured on $11.1 \mathrm{~nm}$ CPG-75 silica glass pores at $T=323 \mathrm{~K}$. Large and small symbols correspond to the large and small simulated pores, respectively.

densities, for $\rho_{\mathrm{b}} / \rho_{\mathrm{c}}>1$, the excess density becomes negative, indicating that the density inside the pore is lower than that in the bulk phase because of steric restrictions arising from inefficient packing under confinement. The higher sorption efficiency of small pores over larger cavities is reflected in $\Delta \rho_{\text {exc }} / \rho_{\text {c }}$ curves, whose values exhibit a twofold increment in going from the wider to the smaller simulated pores. Based on the well-established features of higher fluid storage capacities in narrow cavities, ${ }^{5,27}$ the smaller amplitude of the experimental excess densities as compared to those computed in this work can be ascribed to the difference in pore sizes: whereas the largest pores considered in our MD simulations measured 3.8 $\mathrm{nm}$ in diameter, the experimental curves displayed in Figure 7 were obtained in cavities almost three times larger, that is, in mesoporous controlled pore glass CPG75 of $11.1 \mathrm{~nm}$ size.

We have also examined orientational correlations of the confined molecules. The features of such correlations close to the pore walls can be extracted from the plots depicted in Figure 8, where we examined the following distribution function

$$
P(\cos \theta)=\left\langle\delta\left(\left|\cos \theta_{i}\right|-\cos \theta\right)\right\rangle
$$

where

$$
\cos \theta_{i}=\frac{\mathbf{r} \cdot \hat{\mathbf{u}}_{i}}{|\mathbf{r}|}
$$

In the previous equation, $\hat{\mathbf{u}}_{i}$ represents a unit vector along the molecular axis in the $i$ th molecule. In Figure 8, we present results for $P(\cos \theta)$ for the illustrative case of the equimolar mixture at an intermediate density, $\rho_{\mathrm{b}} / \rho_{\mathrm{c}}=0.46$. In this case, we discriminated three different pore environments: the $5 \AA$ width, outermost adsorption layer, an intermediate $10 \AA \leq r \leq$ $15 \AA ̊$ A cylindrical section, and a bulk-like, $r<10 \AA$, inner region. At first glance, the curves corresponding to interfacial molecules (circles) contrast sharply with the other two. Ethane interfacial species (Figure 8a) presents a maximum for $\cos \theta=$ 0 , indicating parallel alignments with respect to the pore surface. In the intermediate layer (squares), a slight remnant of the propensity to parallel alignments is still perceivable, whereas in the bulk-like region (triangles), the distribution exhibits a flat profile. Interfacial $\mathrm{CO}_{2}$ molecules (Figure 8b), in turn, display a bimodal distribution, with maxima at $\cos \theta=0$ and $\cos \theta=1$, reflecting parallel and perpendicular alignments, with a slight preference for the former arrangements. Within the intermediate layer, $\mathrm{CO}_{2}$ molecules exhibit alignments 

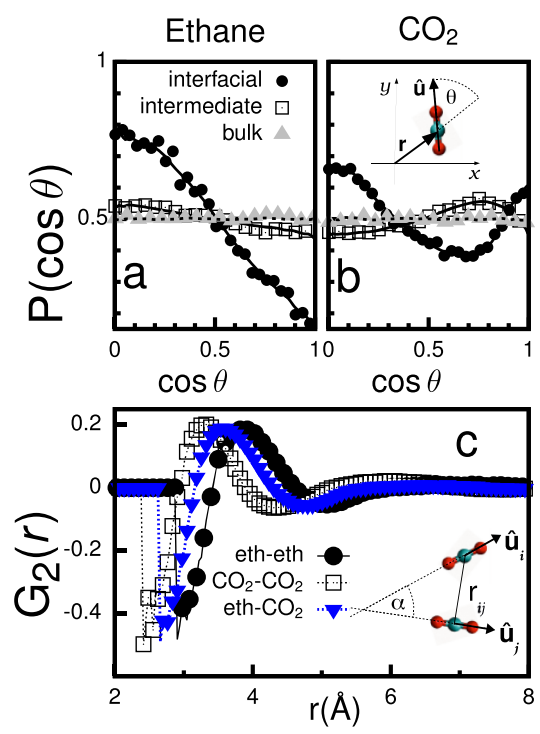

Figure 8. Top panels: Probability distribution $P(\cos \theta)$ as a function of the angle between the molecular axis and the center-of-mass radial vector, for the confined (a) ethane and (b) $\mathrm{CO}_{2}$ molecules located within different regions inside the pores, as indicated. Bottom panel: Legendre polynomials of the cosine angle between pairs of confined molecules, as a function of the center-of-mass distance between them, as indicated. All curves correspond to the equimolar $\mathrm{C}_{2} \mathrm{H}_{6} / \mathrm{CO}_{2}$ mixture with $\rho_{\mathrm{b}} / \rho_{\mathrm{c}}=0.46$.

consistent with angles of $\theta \approx 45^{\circ}$ with respect to the pore walls, that is, midway between parallel and perpendicular orientations. Finally, $\mathrm{CO}_{2}$ molecules in the bulk-like region exhibit a flat structureless profile, as in the case of $\mathrm{C}_{2} \mathrm{H}_{6}$ species.

Perpendicular orientations of $\mathrm{CO}_{2}$ within the adsorption layer were absent in our previous work, ${ }^{27}$ in which the pore walls were modeled as hydrophobic surfaces. In order to show that the change in the overall orientations of $\mathrm{CO}_{2}$ molecules near the surface is not driven by the presence of ethane species, but most likely, by the chemical nature of the pore walls and the stronger fluid/solid interactions, a comparison between the distribution functions $P(\cos \theta)$ for pure $\mathrm{CO}_{2}$ confined within hydrophobic and hydrophilic cavities can be found in the Supporting Information section. A comparative analysis between these two scenarios leads us to infer that it is the presence of the polar $\mathrm{SiOH}$ groups attached to the pore walls that favors the perpendicular $\mathrm{CO}_{2}$ arrangements near the pore surface, induced by the directional hydrogen-bond-like interactions of the type $\mathrm{O}=\mathrm{C}=\mathrm{O} \cdots \mathrm{H}-\mathrm{O}-\mathrm{Si}$. In fact, this has been verified by inspecting a few snapshots of the system. We have identified the $\mathrm{SiOH}-\mathrm{CO}_{2}$ pairs that are forming a hydrogen bond $(\mathrm{HB})$, based on a usual geometric criterion $\left(d(\mathrm{O} \cdots \mathrm{H}) \leq 3.5 \AA\right.$ and $\left.\angle[\mathrm{O} \cdots \mathrm{H}-\mathrm{O}] \geq 120^{\circ}\right)$, and noticed that the $\mathrm{CO}_{2}$ molecules involved in $\mathrm{HBs}$ have perpendicular orientations. We also found a minor fraction of interfacial $\mathrm{CO}_{2}$ molecules that are in perpendicular arrangements but are not explicitly forming HBs. A typical snapshot illustrating these configurations, along with radial pair correlation functions for $\mathrm{O}-\mathrm{O}$ and $\mathrm{O}-\mathrm{H}$ pairs, is provided in the Supporting Information section.

We also looked at the relative orientations between neighboring confined molecules, by computing the following correlation function

$$
G_{2}^{\alpha}(r)=\frac{1}{\left\langle N_{\alpha}\right\rangle_{\mathrm{p}}}\left\langle\sum_{i j} P_{2}\left(\hat{\mathbf{u}}_{i} \cdot \hat{\mathbf{u}}_{j}\right) \delta\left(\left|r_{i j}-r\right|\right)\right\rangle
$$

where $\left\langle N_{\alpha}\right\rangle_{\mathrm{p}}$ accounts for the number of molecules of species $\alpha$ within the pore, $P_{2}(x)=\left(3 x^{2}-1\right) / 2$ is the second-rank Legendre polynomial, $\hat{\mathbf{u}}_{i}$ represents a unit vector along the molecular axis in the $i$-th molecule, and $r_{i j}$ represents the distance between the centers-of-mass of the ith and jth molecules. Reference values of $P_{2}(\cos \theta)=-0.5$ and +1 correspond to perfect T-shaped and parallel configurations, respectively. Results for $G_{2}(r)$ are displayed in Figure 8c, for ethane and $\mathrm{CO}_{2}$ species and cross ethane $/ \mathrm{CO}_{2}$ pairs, in the equimolar system at intermediate density. All these correlations look negative at short distances, denoting a preference for $\mathrm{T}$-shaped arrangements, as found in previous work for pure $\mathrm{CO}_{2}$ confined within hydrophobic pores. ${ }^{27}$ At intermediate separations, $G_{2}(r)$ approaches positive values close to 0.2 , which reveal that, on average, the molecular axes are oriented at an angle of $47^{\circ}$, midway between T-shaped and slid-parallel arrangements. In spite of the fivefold relative difference in the quadrupole moments of ethane and $\mathrm{CO}_{2}$ molecules, the three curves of Figure $8 c$ exhibit very similar shapes and features. In a recent work, Gimondi and Salvalaglio ${ }^{42}$ investigated the effects of cylindrical confinement on $\mathrm{CO}_{2}$ phase transitions. According to their analysis and taking into account the pore size, fluid/wall interactions and fluid densities sampled in the present work, it is possible to characterize the systems under investigation here as disordered phases. In this disordered state,

Table 2. Self Diffusion Coefficients (in $10^{-4} \mathrm{~cm}^{2} \mathrm{~s}^{-1}$ ) of Ethane and $\mathrm{CO}_{2}$ Species in Bulk and Confined Phases within the Wide and Small Pores of Radii $R_{\mathrm{p}}(1)=19 \AA$ and $R_{\mathrm{p}}(2)=5 \AA$, for Pure Ethane Systems and Equimolar Mixtures of Ethane and $\mathrm{CO}_{2}$, at $T=320 \mathrm{~K}^{a}$

\begin{tabular}{|c|c|c|c|c|c|c|c|c|c|}
\hline \multirow[b]{3}{*}{$P$ (bar) } & \multicolumn{3}{|c|}{ pure ethane } & \multicolumn{6}{|c|}{ mixture $50 \% \mathrm{CO}_{2}$} \\
\hline & & \multicolumn{2}{|c|}{$D_{\text {eth }}^{\text {conf }}$} & \multirow[b]{2}{*}{$D_{\text {eth }}^{\mathrm{blk}}$} & \multicolumn{2}{|c|}{$D_{\text {eth }}^{\text {conf }}$} & \multirow[b]{2}{*}{$D_{\mathrm{CO}_{2}}^{\mathrm{blk}}$} & \multicolumn{2}{|c|}{$D_{\mathrm{CO}_{2}}^{\text {conf }}$} \\
\hline & $D_{\text {eth }}^{\mathrm{blk}}$ & $R_{\mathrm{p}}(1)$ & $R_{\mathrm{p}}(2)$ & & $R_{\mathrm{p}}(1)$ & $R_{\mathrm{p}}(2)$ & & $R_{\mathrm{p}}(1)$ & $R_{\mathrm{p}}(2)$ \\
\hline 20 & 20.8 & 2.71 & & 24.2 & 2.88 & & 24.6 & 2.06 & \\
\hline 40 & $7.44[6.7]$ & 2.25 & 0.21 & 8.85 & 2.64 & 0.20 & 8.88 & 1.83 & 0.30 \\
\hline 57 & $6.99[5.8]$ & 1.88 & & 8.86 & 2.38 & & 8.96 & 1.59 & \\
\hline 70 & $4.91[5.3]$ & 1.85 & & 7.67 & 2.02 & & 7.74 & 1.43 & \\
\hline 100 & $3.15[4.0]$ & 1.43 & 0.14 & 3.79 & 1.80 & 0.17 & 3.84 & 1.22 & 0.27 \\
\hline 400 & $1.60[2.1]$ & 1.15 & & 1.51 & 1.98 & & 1.55 & 0.84 & \\
\hline
\end{tabular}

${ }^{a}$ When available, experimental values are also listed between square brackets (refs $\left.43-45\right)$. 
the distribution of relative orientations lies close to the ideal random distribution of linear molecules in the fluid phase, ${ }^{42}$ characterized by the analytic expression: $P_{\text {id }}(\alpha)=1 / 2 \sin \alpha$. This function has a maximum at $\alpha=90^{\circ}$ that corresponds to the perfect T-shaped configuration, regardless of the electrostatic moments of the molecules. Our results for the relative molecular orientations, shown in Figure 8c, are then consistent with the findings reported in ref 42 . We observe in Figure 8c that the location of the minima and maxima do differ for the three curves: whereas the closest approach for ethane pairs is about $3 \AA$, that of $\mathrm{CO}_{2}$ pairs is the smallest, attaining $2.4 \AA$; the closest approach for ethane/ $\mathrm{CO}_{2}$ cross pairs lies intermediate between them, at $2.7 \AA$. This observation is consistent with the larger size of ethane molecules, as revealed by the parameters of the 12-6 Lennard-Jones potential: the corresponding $\sigma$ value of ethane species is $20 \%$ larger than that of $\mathrm{CO}_{2}$ ones. In turn, the values of $\sigma$ reflect the stronger dipolar interactions among $\mathrm{CO}_{2}$ species. Similar shifts can be observed in the location of the global maxima at intermediate separations.

3.2. Dynamical Properties. The translational dynamics of the liquid may be quantitatively evaluated by computing selfdiffusion coefficients from the classical Einstein relation

$$
D=\lim _{t \rightarrow \infty} \frac{\mathcal{R}^{2}(t)}{2 \mathrm{~d} t}=\lim _{t \rightarrow \infty} \frac{\left\langle\left[\mathbf{r}_{i}(t)-\mathbf{r}_{i}(0)\right]^{2}\right\rangle}{2 \mathrm{~d} t}
$$

where $r_{i}(t)$ represents the center-of-mass position of molecule $i$ at time $t, d$ denotes the dimensionality of the system, and $\mathcal{R}^{2}(t)$ is the ensemble-averaged mean squared displacement (MSD). Results for diffusion coefficients of bulk and confined species are reported in Table 2. The former were computed in three dimensions, whereas for the species confined within the cylindrical pore, the diffusion was evaluated along the axial $z$ direction. The bulk values were obtained considering only the molecules at the reservoirs, within the bulk-like region, that is, those located at least $2 \mathrm{~nm}$ away from the pore entrances $(70 \AA$ $\left.<\left|z_{i}\right|<100 \AA\right)$. The diffusion coefficients of the confined fluids were evaluated on modified systems: the lateral reservoir cells in the regions outside the pore were removed, and because of periodic boundary conditions, the cylindrical silica pores became effectively infinite along the $z$-direction. The dimension along the axial direction of the new simulation boxes were then set to $L_{z}=53 \AA$, whereas the other two, $L_{x}$ and $L_{y}$, remained unchanged.

The obtained diffusion coefficients for bulk ethane are in reasonable agreement with experimental works, ${ }^{44,46}$ exhibiting a one-order reduction as going from the lowest to the highest sampled pressures ( $P=20$ bar to $P=400$ bar). The selfdiffusion coefficient values of confined ethane are notably much lower than those of the corresponding bulk phases, show smaller variations with pressure, and decrease monotonically from $2.71 \times 10^{-4} \mathrm{~cm}^{2} \mathrm{~s}^{-1}$ at $P=20$ bar to $1.15 \times 10^{-4} \mathrm{~cm}^{2} \mathrm{~s}^{-1}$ at $P=400$ bar. Experimental measurements of the ratio $D_{\text {eth }}^{\text {blk }} / D_{\text {eth }}^{\text {conf }}$ lie close to $\sim 4$ for pressures within the range $P=$ 38-80 bar, whereas from our MD simulations, it lies between 2 and 3 at similar pressures. In the limit of high pressures, under conditions such that ethane densities inside and outside the silica pore are similar, we observe that diffusion coefficients for bulk and confined ethane seem to converge to a similar low value, around $\sim 1 \times 10^{-4} \mathrm{~cm}^{2} \mathrm{~s}^{-1}$, as a result of a very hindered translational mobility. In the case of the smaller pores, confinement promotes an even more dramatic reduction of the molecular translational mobility along $z$, leading to ethane diffusion coefficients of the order of $10^{-5} \mathrm{~cm}^{2} \mathrm{~s}^{-1}$, representing a reduction by a factor of $\sim 20-35$ with respect to the corresponding bulk values.

At this point, it is worth mentioning that the trends for ethane diffusion as a function of pressure presented here do not agree with the experimental features reported by Patankar et al. ${ }^{15}$ for ethane in mesoporous pore glass silica. These authors found a nonmonotonic behavior of $D_{\text {eth }}^{\text {conf }}$ versus $\rho_{\mathrm{b}}$. In fact, they found that the diffusion of confined ethane decreases with the bulk density (or pressure) at low densities, until $\rho_{\mathrm{b}} / \rho_{\mathrm{c}} \approx 0.75$; further increments in the bulk density induced faster diffusivities. The interpretation of the authors for these features relies on that at low densities, the majority of the fluid molecules accumulate at the solid surface, on high-energy adsorption sites, leading to a reduction in $D_{\text {eth }}^{\text {conf values. At }}$ densities above $\rho_{\mathrm{b}} / \rho_{\mathrm{c}} \approx 0.75$, as all high-energy adsorption sites are already occupied, adsorption occurs on lower-energy sites. Beyond this point, incorporation into the bulk phase starts competing with adsorption into the surface, leading to a faster overall diffusion above that threshold. This behavior is not observed in our simulations. Additionally, Krekelberg et $\mathrm{al}^{21}$ performed an analysis of the relation between structure and dynamics of simple fluids confined in smooth, highly attractive cylindrical pores, with no preferential adsorption sites. Their simulation results are in qualitative agreement with those presented here. Also, simulation results obtained by Le et $\mathrm{al}^{22}$ on propane confined in hydroxylated silica slit-shaped pores are in line with our results, exhibiting a decreasing trend in the diffusivities as the pressure is incremented.

The incorporation of $\mathrm{CO}_{2}$ into the fluid bulk systems enhances the diffusion of ethane species, as revealed by the values listed in Table 2. Except at the highest simulated pressure, an increment of around $20-50 \%$ can be noticed in the diffusion values of ethane species in the bulk mixtures as compared to those corresponding to the pure-ethane bulk systems (columns 2 and 5 of Table 2). A somewhat more moderate increment of ethane diffusion in ethane $/ \mathrm{CO}_{2}$ mixtures has also been reported from experiments. ${ }^{15}$

If we now compare the diffusion coefficients of confined ethane in the pure and ethane $/ \mathrm{CO}_{2}$ mixed systems (columns 3 and 5 in Table 2), we observe that ethane species diffuse faster in the presence of $\mathrm{CO}_{2}$. Moreover, the diffusion coefficients of trapped $\mathrm{CO}_{2}$ species in the mixture are notably slower than those of trapped ethane ones. As discussed in previous sections, the interactions between the silica walls and $\mathrm{CO}_{2}$ are stronger than the ethane/wall ones. Therefore, the incorporation of $\mathrm{CO}_{2}$ would give rise to exchange of ethane molecules from the adsorption layer toward more internal regions within the pore, hand-in-hand with an increment in the relative content of $\mathrm{CO}_{2}$ near the silica walls. As a consequence, the overall mobility of ethane molecules becomes enhanced. These features are better illustrated in Figure 9, where the ratios $D_{\alpha}^{\text {conf }} / D_{\alpha}^{\text {blk }}$ are displayed for ethane and $\mathrm{CO}_{2}$ in the pure and mixed fluids. Note that the curves associated to ethane in the pure phase (squares) and $\mathrm{CO}_{2}$ in the mixture (triangles) exhibit similar characteristics, revealing strong confinement effects at low pressures and a slow increasing trend toward $D_{\alpha}^{\text {conf }} / D_{\alpha}^{\mathrm{blk}} \approx 1$ as long as the pressure is incremented. In contrast, the curve corresponding to ethane in the mixture (circles) reaches values above unity at the largest simulated pressure. This can be rationalized in terms of the ethane density features displayed in Figure 3. In fact, because of the preferential adsorption of $\mathrm{CO}_{2}$ species over ethane ones, see 


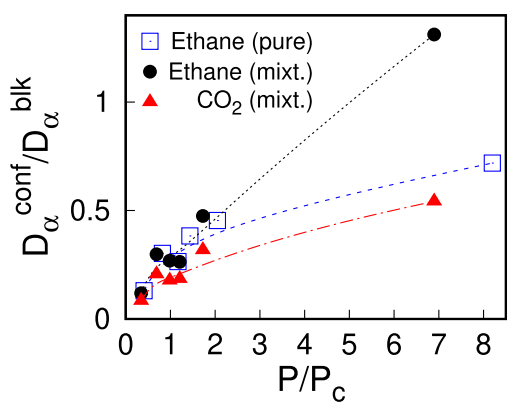

Figure 9. Ratio of confined-to-bulk diffusion coefficients of ethane and $\mathrm{CO}_{2}$ molecules within the mixtures, as a function of pressure. Results for the pure-ethane systems are also displayed for comparison.

for instance Figure 3e, at the highest simulated pressure, the density of ethane inside the pore becomes slightly lower than that in the bulk reservoirs. This small reduction in the density of confined ethane would be sufficient to produce an increment of the translational mobility of molecules as compared to that in the bulk-like environment. Moreover, the radial profiles displayed in Figure 5a show that, at the highest density, there is a substantial increment in the fraction of ethane molecules at the central region inside the pore. As the fluid/wall interactions become negligible within this region, the dynamics of the molecules seems to be dominated not only by thermal but also by density fluctuations. Similar enhancements in the dynamics of hydrocarbons confined in porous environments with the addition of $\mathrm{CO}_{2}$ have been reported in previous works. ${ }^{23,47-49}$

Within the smaller pores, the diffusion coefficients of ethane drop down even more than in the wider pores. The diffusion coefficients are 1 order of magnitude smaller than the values obtained in the wider pores. In this scenario of extreme confinement, the addition of $\mathrm{CO}_{2}$ has barely no effect on ethane mobility at $P=40 \mathrm{bar}$, whereas at high pressure, the presence of $\mathrm{CO}_{2}$ induces a $\sim 20 \%$ diffusion enhancement of ethane species. In passing, it is worth mentioning that the diffusion coefficients of $\mathrm{CO}_{2}$ species in the smaller pores become also substantially reduced-by a factor of $\sim 5-6-$ with respect to the corresponding values in the wider pores. In this context, it is worth mentioning a recent theoretical work, ${ }^{50}$ in which the authors report a reduction of the propane diffusivity in $1.5 \mathrm{~nm}$ cylindrical nanopores with increasing water loading. The slowdown of propane translational mobility is explained in that work in terms of the formation of water bridges with $\mathrm{HB}$ connectivity, which extend over the crosssectional area of the pore, creating long-lived temporary plugs that block the free flow of propane. Besides the significantly smaller molar fractions of propane used in that work as compared to that employed here for ethane, we emphasize that the presence of $\mathrm{HB}$ interactions makes water a very particular fluid that is capable of establishing fairly stable $\mathrm{HB}$ networks that may extend over large extensions. Although $\mathrm{CO}_{2}$ may be able to form $\mathrm{HBs}$ with other molecules, such as $\mathrm{SiO}_{2}$, the strength and features of $\mathrm{CO}_{2}-\mathrm{CO}_{2}$ interactions are very different to those among water molecules. In fact, when the dimensions of the confinement are reduced down to a few molecular diameters, the microscopic details of the intermolecular interactions gain relevance and become essential to explain the characteristics of transport properties.

The existence of different dynamical scales in the bulk and confined phases is evident from the previous paragraphs; we will now show, qualitatively, that within the confined phase itself, a variety of dynamical modes are also present in the trapped fluid. With this in mind, we examine the panels displayed in Figure 10: they show the contour maps of the

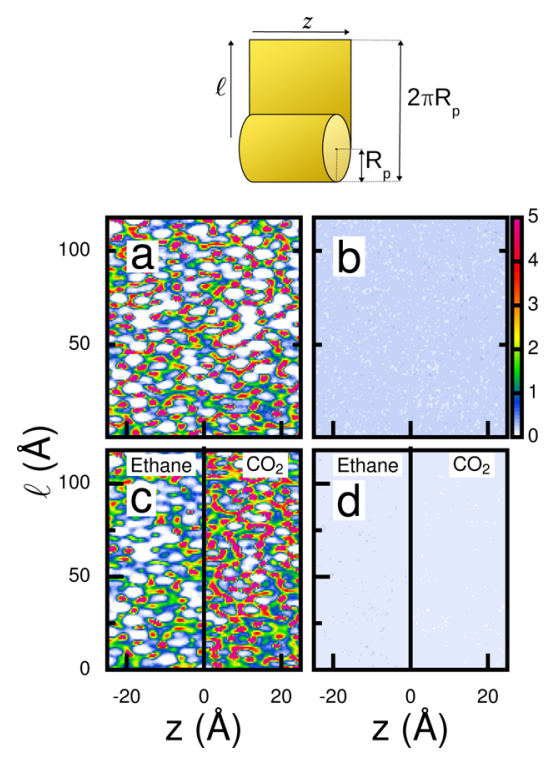

Figure 10. Contour maps of the normalized densities, $\rho(z, l) / \rho_{\mathrm{c}^{\prime}}$ along the lateral unrolled cylindrical surface, for pure ethane $(\mathrm{a}, \mathrm{b})$ and mixtures $(\mathrm{c}, \mathrm{d})$ at $P=40$ bar. Left and right panels correspond to the densities obtained considering the molecules located within the adsorption layer $\left(15 \AA<r<R_{\mathrm{p}}\right)$ and the most central region of the pore $(r<10 \AA)$, respectively.

density along the lateral unrolled cylindrical surface. The abscissa axes of the plots in Figure 10 represent the axial $z$ direction of the cylindrical pores. The polar coordinates of the $i$-th molecule, $\left(x_{i}, y_{i}\right)$, are mapped to the coordinate $l_{i}=R_{\mathrm{p}} \phi_{i}$, represented along the vertical axes in Figure 10, where $\phi_{i}$ is the azimuthal angle

$$
\phi_{i}=\arccos \left\{\frac{x_{i}}{\sqrt{x_{i}^{2}+y_{i}^{2}}}\right\}
$$

We computed the density as

$$
\rho(z, l)=\frac{1}{\Delta r} \sum_{i}\left\langle\delta\left(z_{i}-z\right) \delta\left(l_{i}-l\right)\right\rangle_{\Delta r}
$$

where $\Delta r$ indicates the region considered for the calculation. The densities displayed in Figure 10 were evaluated for $\mathrm{C}_{2} \mathrm{H}_{6}$ and $\mathrm{CO}_{2}$ molecules located at different regions, namely: (i) the outermost layer in contact with the pore walls $\left(15 \AA<r<R_{\mathrm{p}}\right.$, left panels) and (ii) the bulk-like central region of the pore $(r<$ $10 \AA$, right panels). Two low-density representative systems, Eth-40 and Mix-40, were chosen to compute $\rho(z, l)$. At first glance, it is clear that the two sets of results differ at a qualitative level: the most external molecules give rise to a nonuniform density pattern with patches, which are consistent with a strongly adsorbed layer, with hindered mobility. In turn, the overall shape of the patchy structure should be determined by the surface details and rugosity of the solid silica wall. In passing, we notice in panel c-corresponding to the $\mathrm{C}_{2} \mathrm{H}_{6}$ / $\mathrm{CO}_{2}$ mixture-a slightly higher density of $\mathrm{CO}_{2}$ can be appreciated, indicating a greater amount of $\mathrm{CO}_{2}$ adsorbed 
molecules as compared to ethane ones. On the other hand, panels $b$ and $d$, corresponding to the central region, exhibit different features: the density is a uniform distribution, consistent with dynamical features of fluid-like diffusive motions.

By virtue of the coexistence of a variety of dynamical modes in the confined fluid, arising from surface adsorbed and bulklike molecules, we found it appropriate to compute local diffusion coefficients along the axial direction, in terms of the formalism developed by Berne and collaborators. ${ }^{51}$ According to this approach, the MSD is computed in cylindrical concentric layers of width $3 \AA$ A. However, the difficulty when dealing with confined or inhomogeneous systems is that molecules will stay in the region of interest only for a finite time and then will explore other regions. As the diffusion coefficient will be different for different regions, the time dependence of the MSD, computed for particles initially in the region of interest, will become linear only at times long enough for the molecules to sample all regions, and then its slope will give the diffusion coefficient averaged over all regions. Berne and co-workers formulate then the MSD along the axial direction (parallel to the pore surface) of particles that remain in a given region of space as

$$
\mathcal{R}_{z}{ }^{2}(r ; t)=\frac{1}{N(0)} \sum_{i \in \mathcal{S}\{r\}}\left|z_{i}(t)-z_{i}(0)\right|^{2}
$$

where $N(0)$ denotes the number of molecules present in the layer at time zero and $\mathcal{S}\{r\}$ represents the set of molecules that remain continuously in the layer centered at a distance $r$ from the center of the pore, during the interval $[0, t]$. With the above definition for the MSD, molecules that leave the layer and subsequently re-enter are not counted in the summation. The survival probability of molecules in the layer is given by

$$
P(t)=\frac{N(0, t)}{N(0)}
$$

where $N(0, t)$ denotes the number of molecules present in the shell that remain in the same layer for the entire time interval $[0, t]$. Finally, a modified Einstein relation provides the local parallel diffusion coefficient: plotting $\mathcal{R}_{z}{ }^{2}(r ; t) / 2 P(t)$ versus $t$ results in a straight line with a slope equal to the local diffusion coefficient $D(r)$

$$
D(r)=\lim _{t \rightarrow \infty} \frac{\mathcal{R}_{z}^{2}(r ; t)}{2 P(t) t}
$$

We found that the linear diffusive regime of $\mathcal{R}_{z}{ }^{2}(r ; t) / P(t)$ is attained after $\sim 4 \mathrm{ps}$, whereas the typical decay times of the survival functions are of the order of $8-10$ ps. Therefore, the slope is computed in a time interval of around $\sim[4-8] \mathrm{ps}$. The results for ethane local diffusion coefficients are displayed in Figure 11. Plots of the modified MSDs and survival probabilities are provided in the Supporting Information section.

As a general feature, all diffusion coefficients exhibit sharp drops as $r$ approaches the silica walls, regardless of the particular bulk density considered. This observation is consistent with previous studies, ${ }^{52}$ and reveals the coexistence of fast and slow translational modes in the fluid within the pore cavities. Therefore, the values for $D_{\text {eth }}^{\text {conf }}$ listed in Table 2 should be considered as the overall average diffusion inside the pore, taking into account that they are composed of an ensemble of

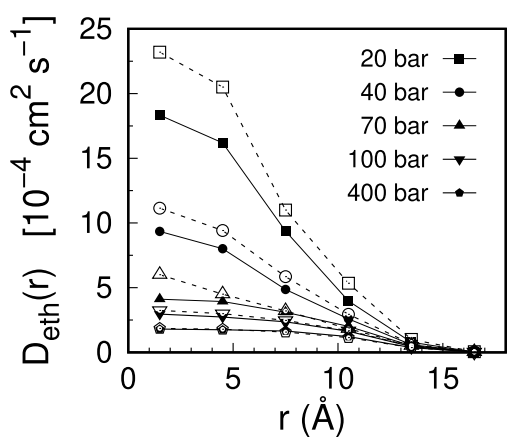

Figure 11. Local diffusion coefficients of confined ethane species, as a function of the distance to the $z$ axis of the cylindrical pore. Results for ethane in the pure systems are represented with black filled symbols; empty symbols correspond to ethane species in equimolar mixtures.

dynamical modes of different timescales. For example, at low pressures, ethane molecules at the central region of the pore may diffuse up to 20 times faster than those in the vicinity of the pore walls. As one approaches the interfacial region, all $D_{\text {eth }}(r)$ profiles approximately collapse onto the same curve, yielding low diffusion coefficients of the order of $\sim 0.5 \times 10^{-4}$ $\mathrm{cm}^{2} \mathrm{~s}^{-1}$. As could be expected, the local diffusion at the central part of the pore becomes comparable to that of the corresponding bulk phases.

The features of the orientational dynamics were analyzed in terms of autocorrelation functions of the type

$$
C_{1}(t)=\left\langle P_{1}\left(\hat{\mathbf{u}}_{i}(t) \cdot \hat{\mathbf{u}}_{i}(0)\right)\right\rangle
$$

where $\hat{\mathbf{u}}_{i}(t)$ represents a unit vector fixed to the molecular axis of molecule $i$ at time $t$, and $P_{1}(x)=x$ is the first-rank Legendre polynomial. The orientational correlation functions were separately computed considering the molecules located within the adsorption layer $(r>15 \AA)$ and within the bulk-like inner region of the pores $(r<10 \AA)$. Figure 12 shows a few selected

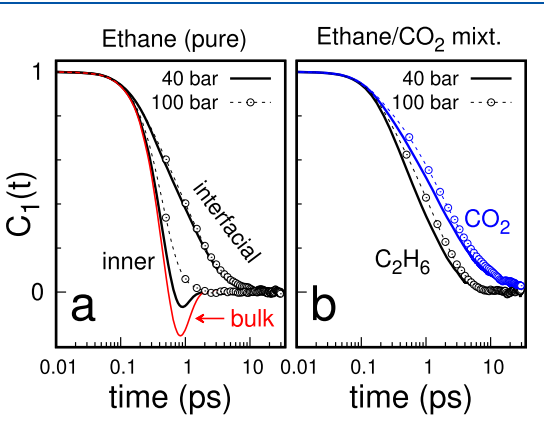

Figure 12. Orientational correlation functions, $C_{1}(t)$, of (a) confined ethane species in pure ethane systems at low and high pressures, as indicated, for molecules located within the interfacial and inner regions inside the pores. For comparison, the curve obtained for pure ethane in the bulk phase is also shown, in red. The curves on panel (b) correspond to interfacial molecules, in ethane $/ \mathrm{CO}_{2}$ confined mixtures at low and high pressures.

representative curves for $C_{1}(t)$ corresponding to low $\left(\rho_{\mathrm{b}} / \rho_{\mathrm{c}} \approx\right.$ $0.3, P=40$ bar $)$ and high $\left(\rho_{\mathrm{b}} / \rho_{\mathrm{c}}>1, P=100 \mathrm{bar}\right)$ fluid densities. In agreement with the retardations found in the diffusive dynamics of ethane as one approaches the silica walls, the orientational correlations plotted in Figure 12a show a pronounced slower decay rate for interfacial ethane compared to the inner molecules, at low and high densities. Moreover, 
whereas the curves pertaining to interfacial molecules at different densities look almost indistinguishable, those associated with the molecules located at the central region do exhibit different features. Namely, at low densities, $C_{1}(t)$ of inner ethane shows a small negative kink at about 1 ps, which would be the signature of librational motions and large-angle reorientations. A comparison with the curve of bulk ethane reveals that these oscillations are remarkably dumped in the confined phase. At higher densities, the overall dynamics becomes more hindered, a slowdown is observed in the decay rate of $C_{1}(t)$, and the kink disappears, leading to a monotonic exponential decay behavior.

On the other hand, Figure $12 \mathrm{~b}$ shows the effect of incrementing the density on the orientational dynamics of interfacial molecules within the mixtures. At first glance it is evident that the reorientational times of ethane species are faster than those of $\mathrm{CO}_{2}$, most likely due to the stronger electrostatic interactions between $\mathrm{CO}_{2}$ and the $\mathrm{SiOH}$ groups attached to the silica walls. We also note that, for both species, increasing the bulk density produces a slight slowing down in the orientational decays.

\section{CONCLUSIONS}

MD simulations were performed to investigate the structural and dynamical properties of fluid ethane confined within cylindrical hydrophilic silica pores of nanometric diameters. Ethane densities in the range $\rho_{\mathrm{b}} / \rho_{\mathrm{c}}=0.13-2.25$ were simulated, along the supercritical isotherm $T=320 \mathrm{~K}$, close to the critical temperature. The effect of the presence of another specie, $\mathrm{CO}_{2}$, on the structural and dynamical features of ethane species has been investigated in equimolar mixtures of ethane $/ \mathrm{CO}_{2}$ with densities lying in a similar range, $\rho_{\mathrm{b}} / \rho_{\mathrm{c}}=$ $0.05-2.18$, along the isotherm $T=320 \mathrm{~K}$.

In agreement with previous experimental data reported for ethane and $\mathrm{CO}_{2}{ }^{15}$ and other simple fluids, ${ }^{5-7,9,53-55}$ we found that the confined phase exhibits a dense adsorbed layer of higher density than the bulk fluid. The excess densities in the single-component fluids exhibit a maximum at low bulk densities, in agreement with experimental observations. At low bulk densities, the average density of the confined fluid may exhibit up to a fivefold increment with respect to that of the bulk phase in the wider pores. The relative density increment is further enhanced in the smaller, $1 \mathrm{~nm}$ pores, up to a factor of 10 .

Radial density profiles revealed the presence of a strongly adsorbed layer near the interface. Ethane molecules in the first layer are mainly oriented parallel to the silica surface, whereas $\mathrm{CO}_{2}$ species may be found in either parallel or perpendicular arrangements, with a small preference for the former orientations over perpendicular ones. Because of the stronger fluid/pore electrostatic interactions between $\mathrm{CO}_{2}$ species and the $\mathrm{SiOH}$ groups of the silica internal surface, a preferential adsorption of $\mathrm{CO}_{2}$ has been found in the confined equimolar mixtures. Although the mixed systems were generated with a global mole fraction of $\mathrm{CO}_{2}$ of 0.50 , we found that the effective mole fraction of $\mathrm{CO}_{2}$ inside the pore was 0.60 , but may increase to $\sim 0.80$ within the adsorbed layer at the highest bulk density investigated.

The analysis of the dynamics of ethane within the pores revealed that the timescales of translational motions become significantly lengthened when compared to values of the corresponding bulk phases, in agreement with experiments. In contrast with experimental data, which reported an increment in the diffusivity of ethane as the pressure increases, near the critical density, ${ }^{15}$ we found a monotonic reduction in the diffusion coefficient of ethane with increasing bulk density. Possible reasons for this discrepancy have been proposed to understand the different features obtained from experiments and simulations.

The preferential adsorption of $\mathrm{CO}_{2}$ over $\mathrm{C}_{2} \mathrm{H}_{6}$ within the adsorbed layer inside the pores led to significant increments in ethane mobility because of displacements of interfacial ethane molecules toward more internal, bulk-like, locations. The ethane diffusivity was also investigated from a local perspective: the local analysis along the radial coordinate of the pore showed that the retardation effects became markedly stronger at the vicinity of the pore walls. In the case of the smaller pores, as the size of the cavity is of the order of a few molecular sizes, almost all confined molecules may be considered as interfacial, and then the effects of confinement on the dynamics become stronger. Under this condition of extreme confinement, the diffusion coefficients are 1 order of magnitude slower than those in the wider pores, yielding values of around $\sim 2 \times 10^{-5} \mathrm{~cm}^{2} \mathrm{~s}^{-1}$. Orientational dynamics was evaluated through time correlation functions. The results revealed that orientational correlations of interfacial ethane molecules decay at a slower rate and have a weaker sensitivity to pressure than those in the bulk-like region.

Summarizing, the results presented here clearly demonstrate the preferential adsorption of $\mathrm{CO}_{2}$ over ethane in silica nanopores and show, as a dynamical consequence, a higher mobility of ethane confined species as the desorption becomes more important. In this line, our results would support the proposal of considering supercritical $\mathrm{CO}_{2}$ as an interesting alternative to water as fracking fluid in shale gas extraction. ${ }^{56,57}$

\section{ASSOCIATED CONTENT}

\section{S Supporting Information}

The Supporting Information is available free of charge at https://pubs.acs.org/doi/10.1021/acs.jpcc.9b07969.

Local densities along $z$ within the smaller pores and in units of $\mathrm{g} \mathrm{cm}^{-1}$, orientational correlations in hydrophobic versus hydrophilic pores, modified mean square displacements in the axial direction, and survival probabilities (PDF)

\section{AUTHOR INFORMATION}

\section{Corresponding Author}

*E-mail: DoloresElola@gmail.com.

ORCID

M. Dolores Elola: 0000-0001-5014-190X

Notes

The authors declare no competing financial interest.

\section{ACKNOWLEDGMENTS}

M.D.E. and J.R. thank ANPCyT (grant PICT 2018-1149) and CONICET (grant PIP 1122015 01-00417) for financial support. M.D.E. and J.R. are staff members of CONICET, Argentina.

\section{REFERENCES}

(1) Steriotis, T. A.; Stefanopoulos, K. L.; Mitropoulos, A. C.; Kanellopoulos, N. K.; Hoser, A.; Hofmann, M. Structural Studies of Supercritical Carbon Dioxide in Confined Space. Appl. Phys. A 2002, 74, S1333-S1335. 
(2) Rother, G.; Melnichenko, Y. B.; Cole, D. R.; Frielinghaus, H.; Wignall, G. D. Mocrostructural Characterization of Adsorption and Depletion Regimes of Supercritical Fluids in Nanopores. J. Phys. Chem. C 2007, 111, 15736-15742.

(3) Belmabkhout, Y.; Sayari, A. Effect of Pore Expansion and Amine Functionalization of Mesoporous Silica on $\mathrm{CO}_{2}$ Adsorption Over a Wide Range of Conditions. Adsorption 2009, 15, 318-328.

(4) Melnichenko, Y. B.; Mayama, H.; Cheng, G.; Blach, T. Monitoring Phase Behavior of Sub- and Supercritical $\mathrm{CO}_{2}$ Confined in Porous Fractal Silica with 85\% Porosity. Langmuir 2010, 26, 63746379.

(5) Rother, G.; Krukowski, E. G.; Wallacher, D.; Grimm, N.; Bodnar, R. J.; Cole, D. R. Pore Size Effects on the Sorption of Supercritical $\mathrm{CO}_{2}$ in Mesoporous CPG-10 Silica. J. Phys. Chem. C 2012, 116, 917922.

(6) Stefanopoulos, K. L.; Steriotis, T. A.; Katsaros, F. K.; Kanellopoulos, N. K.; Hannon, A. C.; Ramsay, J. D. F. Structural Study of Supercritical Carbon Dioxide Confined in Nanoporous Silica by In-Situ Neutron Diffraction. J. Phys.: Conf. Ser. 2012, 340, 012049.

(7) Rother, G.; Vlcek, L.; Gruszkiewicz, M. S.; Chialvo, A. A.; Anovitz, L. M.; Bañuelos, J. L.; Wallacher, D.; Grimm, N.; Cole, D. R. Sorption Phase of Supercritical $\mathrm{CO}_{2}$ in Silica Aerogel: Experiments and Mesoscale Computer Simulations. J. Phys. Chem. C 2014, 118, $15525-15533$.

(8) Gautam, S. S.; Ok, S.; Cole, D. R. Structure and Dynamics of Confined C-O-H Fluids Relevant to the Subsurface: Application of Magnetic Resonance, Neutron Scattering, and Molecular Dynamics Simulations. Front. Earth Sci. 2017, 5, 43.

(9) Gallego, N. C.; He, L.; Saha, D.; Contescu, C. I.; Melnichenko, Y. B. Hydrogen Confinement in Carbon Nanopores: Extreme Densification at Ambient Temperature. J. Am. Chem. Soc. 2011, 133, 13794-13797.

(10) Ciccariello, S.; Melnichenko, Y. B.; He, L. Phase Behavior of Carbon Dioxide Confined in Silica Aerogel in the Vicinity of the Bulk Critical Point. J. Phys. Chem. C 2011, 115, 22336-22346.

(11) Gautam, S.; Liu, T.; Rother, G.; Jalarvo, N.; Mamontov, E.; Welch, S.; Sheets, J.; Droege, M.; Cole, D. R. Dynamics of Propane in Nanoporous Silica Aerogel: A Quasielastic Neutron Scattering Study. J. Phys. Chem. C 2015, 119, 18188-18195.

(12) Gruszkiewicz, M. S.; Wesolowski, D. J.; Cole, D. R. Thermophysical Properties of Pore-Confined Supercritical $\mathrm{CO}_{2}$ by Vibrating Tube Densimetry. Proceedings, Thirty-Sixth Workshop on Geothermal Reservoir Engineering, 2011.

(13) Siemons, N.; Busch, A. Measurement and Interpretation of Supercritical $\mathrm{CO}_{2}$ Sorption on Various Coals. Int. J. Coal Geol. 2007, 69, 229-242.

(14) Watson, G. C.; Jensen, N. K.; Rufford, T. E.; Chan, K. I.; May, E. F. Volumetric Adsorption Measurements of $\mathrm{N}_{2}, \mathrm{CO}_{2}, \mathrm{CH}_{4}$ and a $\mathrm{CO}_{2}+\mathrm{CH}_{4}$ Mixture on a Natural Chabazite from (5 to 3000) $\mathrm{kPa}$. J. Chem. Eng. Data 2011, 57, 93-101.

(15) Patankar, S.; Gautam, S.; Rother, G.; Podlesnyak, A.; Ehlers, G.; Liu, T.; Cole, D. R.; Tomasko, D. L. Role of Confinement on Adsorption and Dynamics of Ethane and Ethane- $\mathrm{CO}_{2}$ Mixture in Mesoporous CPG Silica. J. Phys. Chem. C 2016, 120, 4843-4853.

(16) Qin, Y.; Yang, X.; Zhu, Y.; Ping, J. Simulation of Interaction Between Supercritical $\mathrm{CO}_{2}$ Fluid and Modified Silica Surfaces. J. Phys. Chem. C 2008, 112, 12815-12824.

(17) Yang, X.; Xu, Z.; Zhang, C. Molecular dynamics simulation of dense carbon dioxide fluid on amorphous silica surfaces. J. Colloid Interface Sci. 2006, 297, 38-44.

(18) Yang, X.; Yue, X. Adsorption and Structure of Lennard-Jones Model Fluid in Slit-Like Amorphous Silica nanopores. Colloids Surf., A 2007, 301, 166-173.

(19) Ho, L. N.; Clauzier, S.; Schuurman, Y.; Farrusseng, D.; Coasne, B. Gas Uptake in Solvents Confined in Mesopores: Adsorption Versus Enhanced Solubility. J. Phys. Chem. Lett. 2013, 4, 2274-2278.

(20) Coasne, B.; Pellenq, R. J.-M. Grand canonical Monte Carlo simulation of argon adsorption at the surface of silica nanopores:
Effect of pore size, pore morphology, and surface roughness. J. Chem. Phys. 2004, 120, 2913-2922.

(21) Krekelberg, W. P.; Siderius, D. W.; Shen, V. K.; Truskett, T. M.; Errington, J. R. Connection between Thermodynamics and Dynamics of Simple Fluids in Highly Attractive Pores. Langmuir 2013, 29, 14527-14535.

(22) Le, T.; Striolo, A.; Cole, D. R. Propane Simulated in Silica Pores: Adsorption Isotherms, Molecular Structure, and Mobility. Chem. Eng. Sci. 2015, 121, 292-299.

(23) Le, T.; Striolo, A.; Cole, D. R. $\mathrm{CO}_{2}-\mathrm{C}_{4} \mathrm{H}_{10}$ Mixtures Simulated in Silica Slit Pores: Relation between Structure and Dynamics. J. Phys. Chem. C 2015, 119, 15274-15284.

(24) Gautam, S.; Liu, T.; Cole, D. Sorption, Structure and Dynamics of $\mathrm{CO}_{2}$ and Ethane in Silicalite at High Pressure: A Combined Monte Carlo and Molecular Dynamics Simulation Study. Molecules 2019, 24, 99.

(25) de Luca, G.; Russo, N.; Sicilia, E.; Toscano, M. Molecular Quadrupole Moments, Second Moments, and Diamagnetic Susceptibilities Evaluated Using the Generalized Gradient Approximation in the Framework of Gaussian Density Functional Method. J. Chem. Phys. 1996, 105, 3206-3210.

(26) Junquera-Hernández, J. M.; Sánchez-Marín, J.; Maynau, D. Molecular electric quadrupole moments calculated with matrix dressed SDCI. Chem. Phys. Lett. 2002, 359, 343-348.

(27) Elola, M. D.; Rodriguez, J. Excess Sorption of Supercritical $\mathrm{CO}_{2}$ within Cylindrical Silica Nanopores. J. Phys. Chem. C 2016, 120, $1262-1269$

(28) Shah, M. S.; Siepmann, J. I.; Tsapatsis, M. Transferable Potentials for Phase Equilibria. Improved United-Atom Description of Ethane and Ethylene. AIChE J. 2017, 63, 5098-5110.

(29) Ambrose, D.; Tsonopoulos, C. Vapor-Liqud Critical Properties of Elements and Compounds, 2: Normal Alkanes. J. Chem. Eng. Data 1995, 40, 531-546.

(30) Harris, J. G.; Yung, K. H. Carbon Dioxide's Liquid-Vapor Coexistence Curve and Critical Properties as Predicted by a Simple Molecular Model. J. Phys. Chem. 1995, 99, 12021-12024.

(31) Abbaci, A.; van den Berg, H. R.; Sakonidou, E.; Sengers, J. V. Critical Parameters of Mixtures of Carbon Dioxide and Ethane. Int. J. Thermophys. 1992, 13, 1043-1052.

(32) Rodriguez, J.; Elola, M. D.; Laria, D. Confined Polar Mixtures Within Cylindrical Nanocavities. J. Phys. Chem. B 2010, 114, 79007908.

(33) Elola, M. D.; Rodriguez, J.; Laria, D. Structure and Dynamics of Liquid Methanol Confined Within Functionalized Silica Nanopores. J. Chem. Phys. 2010, 133, 154707-154715.

(34) Elola, M. D.; Rodriguez, J.; Laria, D. Liquid Methanol Confined within Functionalized Silica Nanopores. 2. Solvation Dynamics of Coumarin 153. J. Phys. Chem. B 2011, 115, 12859-12867.

(35) Kamijo, T.; Yamaguchi, A.; Suzuki, S.; Teramae, N.; Itoh, T.; Ikeda, T. Solvation Dynamics of Coumarin 153 in Alcohols Confined in Silica Nanochannels. J. Phys. Chem. A 2008, 112, 11535-11542.

(36) Lemmon, E. W.; McLinden, M. O.; Friend, D. G. NIST Chemistry WebBook; Linstrom, P. J., Mallard, W. G., Eds.; NIST Standard Reference Database Number 69; NIST: Gaithersburg MD, USA, 2019; Chapter Thermophysical Properties of Fluid Systems.

(37) Phillips, J. C.; Braun, R.; Wang, W.; Gumbart, J.; Tajkhorshid, E.; Villa, E.; Chipot, C.; Skeel, R. D.; Kalé, L.; Schulten, K. Scalable Molecular Dynamics with NAMD. J. Comput. Chem. 2005, 26, 17811802.

(38) Darden, T.; York, D.; Pedersen, L. Particle Mesh Ewald - An N. $\log (\mathrm{N})$ Method for Ewald Sums in Large Systems. J. Chem. Phys. 1993, 98, 10089.

(39) Essmann, U.; Perera, L.; Berkowitz, M. L.; Darden, T.; Lee, H.; Pedersen, L. G. A Smooth Particle Mesh Ewald Method. J. Chem. Phys. 1995, 103, 8577.

(40) Tuckerman, M.; Berne, B. J.; Martyna, G. J. Reversible Multiple Time Scale Molecular Dynamics. J. Chem. Phys. 1992, 97, 19902001. 
(41) Grubmüller, H.; Heller, H.; Windemuth, A.; Schulten, K. Generalized Verlet Algorithm for Efficient Molecular Dynamics Simulations with Long-range Interactions. Mol. Simul. 1991, 6, 121-142.

(42) Gimondi, I.; Salvalaglio, M. $\mathrm{CO}_{2}$ Packing Polymorphism under Confinement in Cylindrical Nanopores. Mol. Syst. Des. Eng. 2018, 3, $243-252$.

(43) Hara, K.; Ito, N.; Kajimoto, O. High Pressure Studies of the Kramers Turnover Behavior for the Excited-State Isomerization of 2Alkenylanthracene in Alkane. J. Chem. Phys. 1999, 110, 1662-1668.

(44) Adam, W.; Diedering, M.; Trofimov, A. V. Pressure Dependence of the Stereoselectivity in the Photodenitrogenation of Diazabicyclo[2.2.1] hept-2-ene in Supercritical Fluids: Evidence for the Diazenyl Diradical. Chem. Phys. Lett. 2001, 350, 453-458.

(45) Helbæk, M.; Hafskjold, B.; Dysthe, D. K.; Sørland, G. H. SelfDiffusion Coefficients of Methane or Ethane Mixtures with Hydrocarbons at High Pressure by NMR. J. Chem. Eng. Data 1996, 41, 598-603.

(46) Greiner-Schmid, A.; Wappmann, S.; Has, M.; L udemann, H. D. Self-Diffusion in the Compressed Fluid Lower Alkanes: Methane, Ethane, and Propane. J. Chem. Phys. 1991, 94, 5643-5649.

(47) Chathoth, S. M.; He, L.; Mamontov, E.; Melnichenko, Y. B. Effect of Carbon Dioxide and Nitrogen on the Diffusivity of Methane Confined in Nano-porous Carbon Aerogel. Microporous Mesoporous Mater. 2012, 148, 101-106.

(48) Yang, N.; Liu, S.; Yang, X. Molecular Simulation of Preferential Adsorption of $\mathrm{CO}_{2}$ over $\mathrm{CH}_{4}$ in Na-montmorillonite Clay Material. Appl. Surf. Sci. 2015, 356, 1262-1271.

(49) Salles, F.; Jobic, H.; Devic, T.; Guillerm, V.; Serre, C.; Koza, M. M.; Ferey, G.; Maurin, G. Diffusion of Binary $\mathrm{CO}_{2} / \mathrm{CH}_{4}$ Mixtures in the MIL-47(V) and MIL-53(Cr) Metal-Organic Framework Type Solids: A Combination of Neutron Scattering Measurements and Molecular Dynamics Simulation. J. Phys. Chem. C 2013, 117, 1127511284 .

(50) Le, T. T. B.; Striolo, A.; Gautam, S. S.; Cole, D. R. PropaneWater Mixtures Confined within Cylindrical Silica Nanopores: Structural and Dynamical Properties Probed by Molecular Dynamics. Langmuir 2017, 33, 11310-11320.

(51) Liu, P.; Harder, E.; Berne, B. J. On the Calculation of Diffusion Coefficients in Confined Fluids and Interfaces with an Application to the Liquid-Vapor Interface of Water. J. Phys. Chem. B 2004, 108, 6595-6602.

(52) Castrillon, S. R.-V.; Giovambattista, N.; Aksay, I. A.; Debenedetti, P. G. Effect of Surface Polarity on the Structure and Dynamics of Water in Nanoscale Confinement. J. Phys. Chem. B 2009, 113,1438

(53) Lastoskie, C. Caging Carbon Dioxide. Science 2010, 330, 595596.

(54) Vaidhyanathan, R.; Iremonger, S. S.; Shimizu, G. K. H.; Boyd, P. G.; Alavi, S.; Woo, T. K. Direct Observation and Quantification of $\mathrm{CO}_{2}$ Binding Within an Amine-Functionalized Nanoporous Solid. Science 2010, 330, 650-653.

(55) Do, D. D.; Do, H. D. Adsorption of argon from sub- to supercritical conditions on graphitized thermal carbon black and in graphitic slit pores: A grand canonical Monte Carlo simulation study. J. Chem. Phys. 2005, 123, 084701.

(56) Pei, P.; Ling, K.; He, J.; Liu, Z. Shale Gas Reservoir Treatment by a $\mathrm{CO}_{2}$-based Technology. J. Nat. Gas Sci. Eng. 2015, 26, 15951606.

(57) Middleton, R. S.; Carey, J. W.; Currier, R. P.; Hyman, J. D.; Kang, Q.; Karra, S.; Jiménez-Martínez, J.; Porter, M. L.; Viswanathan, H. S. Shale Gas and Non-aqueous Fracturing Fluids: Opportunities and Challenges for Supercritical $\mathrm{CO}_{2}$. Appl. Energy 2015, 147, 500509. 